\title{
Rapid development of a tophus following ipsilateral hemiparesis
}

\author{
Ciaran F Donegan, Peter Berman, Michael Doherty
}

\begin{abstract}
An 85 year old man with a longstanding history of non-tophaceous gout developed a single large tophus, unassociated with inflammation, on his paretic leg over a six week period following an acute hemiplegia. The rapidity of tophus formation, its localisation to the paretic limb, and the apparent blunting of the acute inflammatory response represent a previously unreported interaction between gout and neurological deficit.
\end{abstract}

(Ann Rheum Dis 1993; 52: 308-309)

Considerable interest exists in the interaction between neurological deficits and the clinical expression of rheumatic diseases. The sparing effect of upper or lower neurological deficit on the subsequent development of arthropathy, including gout, has been well described. ${ }^{1-6}$ Conversely, a few cases have been described in which exacerbation of established joint disease has followed hemiparesis. ${ }^{7-9}$ We report an apparently unique patient with a history of recurrent acute gout, in whom the development of a hemiparesis was followed by the rapid appearance of a non-inflammatory tophus on the heel of his paretic foot.

\section{Case report}

A previously fit 85 year old right handed man was admitted with an acute left hemiplegia. Since the age of 50 he had had recurrent acute attacks of gout limited to both feet (including classic podagra), treated with short intermittent courses of non-steroidal antiinflammatory drugs and allopurinol. He had not taken such drugs for many months. There were no associated risk factors for gout (obesity, excess alcohol, chronic diuretic treatment, family history) and he had never developed tophi. Examination confirmed marked left sided weakness affecting the arm more than the leg, mild sensory signs but no visual field deficit. A computed tomography brain scan showed right frontoparietal infarction.

Over the subsequent six weeks his motor function improved, though he still required the assistance of one person for most activities. At this time he complained of a swelling over his left heel which prevented him from wearing his normal footwear. The swelling, at the level of the pre-Achilles bursa, was $2 \times 3 \mathrm{~cm}$, firm, and non-tender. The overlying skin was healthy and intact with no signs of local inflammation. No other swellings were apparent elsewhere. A lateral radiograph (fig 1) showed soft tissue swelling superficial to the Achilles tendon insertion with an underlying small focus of calcification. Aspiration of the swelling confirmed urate crystals in a typical tophus 'sheet' configuration (compensated polarised light microscopy). After aspiration macroscopically white crystal deposits exuded through the needle track (fig 2), without provoking an inflammatory response.

Further investigations showed: urea 11.7 $\mathrm{mmol} / \mathrm{l}$ (normal range 1-6.5); creatinine 167 $\mu \mathrm{mol} / 1 \quad(60-120)$; uric acid $406 \mu \mathrm{mol} / 1$ (100-400); calcium and fasting lipoproteins within normal limits. Radiographs of feet, knees, pelvis, and hands showed only minor changes of osteoarthritis (knees, first metatarsophalangeal joints) and no chondrocalcinosis. Treatment was established with allopurinol $100 \mathrm{mg}$ daily. Because the

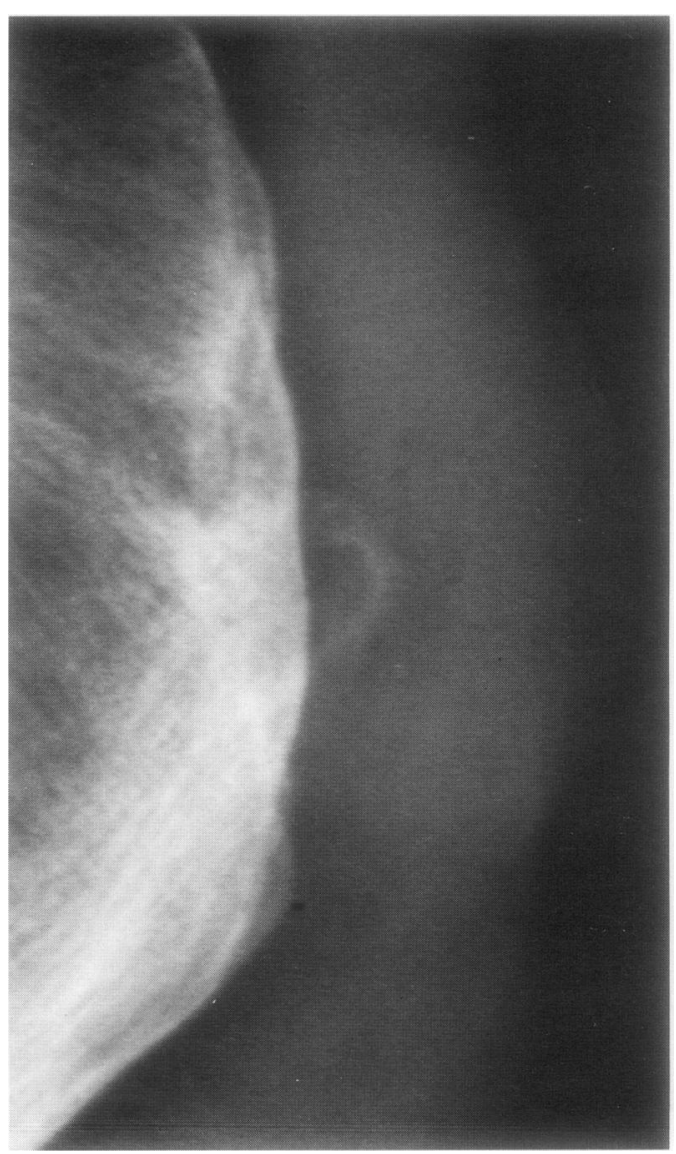

Figure 1 Lateral left heel radiograph showing soft tissue swelling and central area of calcification. 


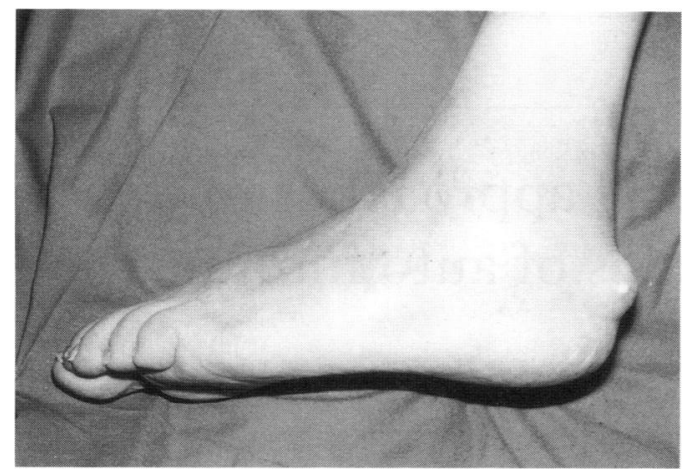

Figure 2 The left heel of the patient following aspiration, showing white tophaceous material that had travelled up the needle track.

mechanical effect of the large tophus was impeding mobilisation it was excised nine weeks after his stroke. His subsequent recovery was uneventful.

\section{Discussion}

To our knowledge this is the first report of a tophus developing in a paretic limb following acute neurological deficit. The close temporal link between hemiparesis and development of the tophus, its ipsilateral location, and the absence of a previous history or evidence of other tophi strongly support a causal association. Two further features of interest are the rapidity of development and the absence of associated inflammation.

Mechanisms to explain exacerbation of arthropathy (rheumatoid arthritis, osteoarthritis) following hemiparesis favour neurovascular alteration of blood flow or altered neurotrophic effects on inflammation. ${ }^{78}$ The converse sparing effect of upper and lower motor neurone lesions on development of locomotor disease (including gout) has more commonly been attributed to disuse with reduction of damaging mechanical factors and intra-articular pressure. ${ }^{24-6}$ Acute self limiting arthropathy has been seen in hemiplegic limbs of patients with no prior history of arthritis shortly after a cerebrovascular accident ${ }^{10}$; although reaction to underlying infection has been incriminated, ${ }^{11}$ the cause remains speculative.

In the present case it is possible that minor tissue damage or unrecognised trauma, consequent upon weakness and sensory deficit, promoted tophus formation in a predisposed patient. Alternatively, altered neurovascular response in a paretic limb following stroke may lead to increased tissue urate concentrations and possibly predispose to the development of tophi. ${ }^{12}$ Although there was no overt preAchilles trauma, ulceration or bursitis, the calcific focus on the radiograph might support local tissue injury and indicate that a small, unidentified (mixed) crystal deposit had been present at this site for some time. Whether a crystal mass originated before or after the stroke, however, the hemiparesis seems to have encouraged accelerated crystal deposition and tophus development.

The only comparable case is that reported by Cosgrave and Lewkonia ${ }^{7}$ of a patient with posttraumatic 'frozen shoulder' who developed tophi in the ipsilateral hand six months later. Although there was no neurological deficit, disuse or altered regional blood flow were again both proposed as mechanisms of causation.

Tophus formation is normally regarded as a slow process. Although in vitro formation of monosodium urate monohydrate crystals can be rapid, in vivo formation is estimated to take many months, ${ }^{13}$ and tophi usually occur in association with symptomatic chronic gout. In our patient the speed of formation of his first ever tophus was rapid. The absence of associated inflammation is also remarkable, particularly as newly forming (compared with older) urate crystals might be expected to expose more 'naked', non-protein coated surfaces for interaction with cell surfaces and inflammatory mediators. ${ }^{14}$ The morphology of the urate crystals seemed typical of those seen in tophi, though it is possible that such rapidly forming crystals might present less active surfaces with respect to surface charge and 'roughness'. Diminution of the inflammatory reaction consequent upon altered neurovascular responses would seem a more plausible explanation, possibly mediated through reduction in neurotransmitters. ${ }^{15}$

1 Glynn J J, Clayton M L. Sparing effect of hemiplegia on tophaceous gout. Ann Rheum Dis 1976; 35: 534-5.

2 Thompson M, Bywaters E G L. Unilateral rheumatoid arthritis following hemiplegia. Ann Rheum Dis 1962; 21: $370-7$.

3 Coste F, Forestier J. Hemiplegie et nodosites d'Heberden contralaterales. Bull Mem Soc Med Hop Paris 1935; 51: $772-6$

4 Glick E N. Asymmetrical rheumatoid arthritis after poliomyelitis. $B M F$ 1967; iii: $26-8$.

5 Glynn J H, Sutherland I, Walker G F, Young A C. Low incidence of osteoarthrosis in hip and knee after anterior poliomyelitis: a late review. BMF 1966; ii: 739-42.

6 Needs C J, Webb J, Tyndall A. Paralysis and unilateral arthritis: is the association established? Clin Rheumatol arthritis: is the

7 Cosgrave P M, Lewkonia R M. Asymmetric tophaceous gout. f Rheumatol 1979; 6: 299-301.

8 Pattrick M, Doherty M, Dieppe P A. Unilateral exacerbation of rheumatoid arthritis by hemiparesis. $\mathrm{Br} \mathcal{F}$ Rheumatol 1984; 23: 107-9.

9 Pattrick M, Doherty $M$. Rapidly destructive hip disease following unilateral hemiparesis: report of two cases. Ann Rheum Dis 1987; 46: 477-81.

10 Hermann E. Acute arthritis in hemiplegics. Scand $f$ Rheumatol 1972; 1: 87-9.

11 Mitchell W S, Capell H A. Unexplained acute arthritis in hemiplegia. BMf 1982; Vol: 284-6.

2 Simkin P. The pathogenesis of podagra. Ann Intern Med 1977; 86: 230-3.

13 Fiddis R W, Vlachos N, Calvert P D. Studies of urate crystallisation in relation to gout. Ann Rheum Dis 1983; 42 (suppl 1); 12-15.

14 Doherty M, Dieppe P A. Crystal deposition disease in the elderly. Clin Rheum Dis 1986; 12: 97-116.

15 Levine J D, Goetzl E J, Basbaum A I. Contribution of the nervous system to the pathophysiology of rheumatoid arthritis and other polyarthritides. Rheum Dis Clin North Am 1987; 13: 369-83. 